\title{
Measurement of an elongation of the pion source in $\mathrm{Z}$ decays
}

\author{
L3 Collaboration
}

M. Acciarri ${ }^{y}$, P. Achard ' , O. Adriani ${ }^{\circ}$, M. Aguilar-Benitez ${ }^{x}$, J. Alcaraz ${ }^{x}$, G. Alemanni ", J. Allaby ${ }^{\text {p }}$, A. Aloisio ${ }^{\text {aa }}$, M.G. Alviggi ${ }^{\text {aa }}$, G. Ambrosi ${ }^{\text {r }}$, H. Anderhub at, V.P. Andreev f,ai, T. Angelescu ', F. Anselmo i, A. Arefiev ${ }^{\mathrm{z}}$, T. Azemoon ${ }^{\text {c }}$, T. Aziz ${ }^{j}$, P. Bagnaia ${ }^{\text {ah }}$, L. Baksay ${ }^{\text {ao }}$, A. Balandras ${ }^{\text {d }}$, R.C. Ball ${ }^{\text {c }}$, S. Banerjee ${ }^{j}$, Sw. Banerjee ${ }^{j}, K$. Banicz ${ }^{\text {aq }}$, A. Barczyk ${ }^{\text {at.ar }}$, R. Barillère ${ }^{p}$, L. Barone ah , P. Bartalini ", M. Basile ${ }^{\text {i }, ~ R . ~ B a t t i s t o n ~}{ }^{\text {ae }}$, A. Bay ", F. Becattini ${ }^{\circ}$, U. Becker ", F. Behner ${ }^{\text {at }}$, J. Berdugo ${ }^{\mathrm{x}}$, P. Berges ${ }^{\mathrm{n}}$, B. Bertucci ${ }^{\text {ae }}$, B.L. Betev ${ }^{\text {at }}$, S. Bhattacharya ${ }^{j}$, M. Biasini ${ }^{\text {ae }}$, A. Biland ${ }^{\text {at }}$, J.J. Blaising ${ }^{\mathrm{d}}$, S.C. Blyth ${ }^{\text {af }}$, G.J. Bobbink ${ }^{\text {b }, ~ A . ~ B o ̈ h m ~}{ }^{\text {a }}$, L. Boldizsar ${ }^{\mathrm{m}}$, B. Borgia ${ }^{\text {ah }}$, D. Bourilkov ${ }^{\text {at }}$, M. Bourquin ' , S. Braccini ${ }^{\mathrm{r}}$, J.G. Branson ${ }^{\text {ak }}$, V. Brigljevic ${ }^{\text {at }}$, F. Brochu ${ }^{\mathrm{d}}$, A. Buffini ${ }^{\circ}$, A. Buijs ${ }^{\text {ap }}$, J.D. Burger ${ }^{\text {n, W.J. Burger }}{ }^{\text {ac }}$, J. Busenitz ${ }^{\text {ao }}$, A. Button ${ }^{\text {c }}$, X.D. Cai ", M. Campanelli at, M. Capell ", G. Cara Romeo i, G. Carlino ${ }^{\text {aa }}$, A.M. Cartacci ${ }^{\circ}$, J. Casaus ${ }^{x}$, G. Castellini ${ }^{\circ}$, F. Cavallari ah, N. Cavallo ${ }^{\text {aa }}$, C. Cecchi ${ }^{r}$, M. Cerrada ${ }^{x}$, F. Cesaroni ${ }^{\text {v }}$, M. Chamizo ${ }^{r}$, Y.H. Chang av, U.K. Chaturvedi ${ }^{\mathrm{q}}$, S.V. Chekanov ${ }^{\text {ac }}$, M. Chemarin ${ }^{\text {w }}$, A. Chen ${ }^{\text {av }}$, G. Chen ${ }^{\mathrm{g}}$, G.M. Chen ${ }^{\text {g }}$, H.F. Chen ${ }^{\text {s, H.S. Chen }}{ }^{\mathrm{g}}$, X. Chereau ${ }^{\mathrm{d}}$, G. Chiefari ${ }^{\text {aa }}$, L. Cifarelli ${ }^{\text {aj, }}$, F. Cindolo ${ }^{\text {i, C. Civinini }}{ }^{\text {, }}$ I. Clare n, R. Clare ", G. Coignet d, A.P. Colijn ", N. Colino ${ }^{x}$, S. Costantini ${ }^{\text {h }}$, F. Cotorobai ', B. Cozzoni i , B. de la Cruz ${ }^{x}$, A. Csilling ${ }^{\mathrm{m}}$, S. Cucciarelli ${ }^{\text {ae }}$, T.S. Dai ${ }^{\mathrm{n}}$, J.A. van Dalen ${ }^{\text {ac }}$, R. D’Alessandro ${ }^{\circ}$, R. de Asmundis ${ }^{\text {aa }}$, P. Deglon ${ }^{\mathrm{r}}$, A. Degré ${ }^{\mathrm{d}}$, K. Deiters ${ }^{\mathrm{ar}}$, D. della Volpe ${ }^{\text {aa }}$, P. Denes ${ }^{\text {ag }}$, F. DeNotaristefani ah, A. De Salvo ${ }^{\text {at }}$, M. Diemoz ${ }^{\text {ah }}$, M. van Dierendonck ${ }^{b}$, F. Di Lodovico ${ }^{\text {at }}$, C. Dionisi ${ }^{\text {ah }}$, M. Dittmar ${ }^{\text {at }}$, A. Dominguez ${ }^{\text {ak }}$, A. Doria ${ }^{\text {aa }}$, M.T. Dova ${ }^{\text {q.1 }}$, D. Duchesneau ${ }^{d}$, D. Dufournand ${ }^{d}$, P. Duinker ${ }^{\text {b }}$, I. Duran ${ }^{\text {al }}$, H. El Mamouni ${ }^{\text {w }}$, A. Engler ${ }^{\text {af }}$, F.J. Eppling ${ }^{\text {n }}$, F.C. Erné ${ }^{b}$, P. Extermann ${ }^{r}$, M. Fabre ${ }^{\text {ar }}$, R. Faccini ${ }^{\text {ah }}$, M.A. Falagan ${ }^{\mathrm{x}}$, S. Falciano ${ }^{\text {ah, }}$, A. Favara $^{\text {p }}$, J. Fay ${ }^{\text {w }}$, O. Fedin ${ }^{\text {ai }}$, M. Felcini ${ }^{\text {at }}$, T. Ferguson ${ }^{\text {af }}$, F. Ferroni ${ }^{\text {ah }}$, H. Fesefeldt ${ }^{a}$, E. Fiandrini ${ }^{\text {ae }}$, J.H. Field ${ }^{r}$, F. Filthaut ${ }^{\text {p }}$, P.H. Fisher ${ }^{n}$, I. Fisk ${ }^{\text {ak }}$, G. Forconi ", L. Fredj ' , K. Freudenreich ${ }^{\text {at }}$, C. Furetta ${ }^{y}$, Yu. Galaktionov ${ }^{\text {z,n }}$, 
S.N. Ganguli ${ }^{\mathrm{j}}$, P. Garcia-Abia ${ }^{\mathrm{e}}$, M. Gataullin ${ }^{\text {ad }}$, S.S. Gau ${ }^{\mathrm{k}}$, S. Gentile ${ }^{\text {ah,p }}$,

N. Gheordanescu ${ }^{1}$, S. Giagu ${ }^{\text {ah }}$, Z.F. Gong s , G. Grenier ${ }^{\text {w }}$, O. Grimm ${ }^{\text {at }}$,

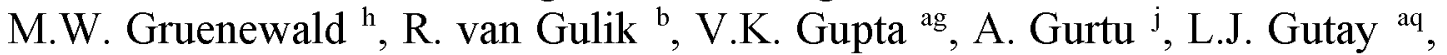
D. Haas ${ }^{e}$, A. Hasan ${ }^{a b}$, D. Hatzifotiadou ${ }^{i}$, T. Hebbeker ${ }^{\text {h}}$, A. Hervé ${ }^{\mathrm{p}}$, P. Hidas ${ }^{\mathrm{m}}$, J. Hirschfelder ${ }^{\text {af }}$, H. Hofer ${ }^{\text {at }}$, G. Holzner ${ }^{\text {at }}$, H. Hoorani ${ }^{\text {af }}$, S.R. Hou ${ }^{\text {av }}$, I. Iashvili as, B.N. Jin ${ }^{\mathrm{g}}$, L.W. Jones ${ }^{\text {c }}$, P. de Jong ${ }^{\mathrm{b}}$, I. Josa-Mutuberría ${ }^{\mathrm{x}}$, R.A. Khan ${ }^{\mathrm{q}}$, D. Kamrad ${ }^{\text {as }}$, M. Kaur ${ }^{\text {q,2 }}$, M.N. Kienzle-Focacci ${ }^{r}$, D. Kim ${ }^{\text {ah }}$, D.H. Kim ${ }^{\text {an }}$, J.K. Kim ${ }^{\text {an }}$, S.C. Kim ${ }^{\text {an }}$, J. Kirkby ${ }^{\text {p }}$, D. Kiss ${ }^{\text {m }}$, W. Kittel ${ }^{\text {ac }}$, A. Klimentov ${ }^{n, z}$, A.C. König ${ }^{\text {ac }}$, A. Kopp ${ }^{\text {as }}$, I. Korolko ${ }^{z}$, V. Koutsenko ${ }^{\text {n,z }}$, M. Kräber ${ }^{\text {at }}$, R.W. Kraemer af , W. Krenz a , A. Kunin ${ }^{n, z}$, P. Lacentre ${ }^{\text {as, }, 1,3}$, P. Ladron de Guevara ${ }^{\mathrm{x}}$,

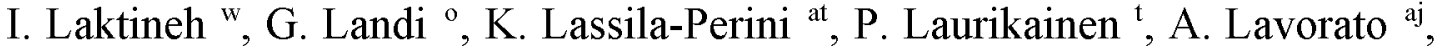
M. Lebeau ${ }^{\mathrm{p}}$, A. Lebedev ${ }^{\mathrm{n}}$, P. Lebrun ${ }^{\mathrm{w}}$, P. Lecomte ${ }^{\text {at }}$, P. Lecoq ${ }^{\mathrm{p}}$, P. Le Coultre ${ }^{\text {at }}$, H.J. Lee ${ }^{\mathrm{h}}$, J.M. Le Goff ${ }^{\mathrm{p}}$, R. Leiste ${ }^{\text {as }}$, E. Leonardi ${ }^{\text {ah }}$, P. Levtchenko ${ }^{\text {ai }}, \mathrm{C}$ Li $^{\mathrm{s}}$, C.H. Lin ${ }^{\text {av }}$, W.T. Lin ${ }^{\text {av }}$, F.L. Linde ${ }^{\text {b }}$, L. Lista ${ }^{\text {aa }}$, Z.A. Liu ${ }^{\mathrm{g}}$, W. Lohmann ${ }^{\text {as }}$, E. Longo ah, Y.S. Lu ${ }^{g}$, K. Lübelsmeyer ${ }^{\text {a }}$, C. Luci ${ }^{\text {pah }}$, D. Luckey ${ }^{\text {n }}$, L. Lugnier ${ }^{\text {w }}$, L. Luminari ah, W. Lustermann ${ }^{\text {at }}$, W.G. Ma ${ }^{\text {s }}$, M. Maity ${ }^{j}$, L. Malgeri ${ }^{\mathrm{p}}$, A. Malinin $^{\text {z,p }}$, C. Maña ${ }^{\mathrm{x}}$, D. Mangeol ${ }^{\text {ac }}$, P. Marchesini ${ }^{\text {at }}$, G. Marian ${ }^{\text {ao, } 4}$, J.P. Martin ${ }^{\text {w }}$, F. Marzano ${ }^{\text {ah }}$, G.G.G. Massaro ${ }^{\text {b }}$, K. Mazumdar ${ }^{j}$, R.R. McNeil ${ }^{\mathrm{f}}$, S. Mele ${ }^{p}$, L. Merola $^{\text {aa }}$, M. Meschini ${ }^{\circ}$, W.J. Metzger ${ }^{\text {ac }}$, M. von der Mey ${ }^{a}$, D. Migani ${ }^{\mathrm{i}}$, A. Mihul ${ }^{1}$, H. Milcent ${ }^{\mathrm{p}}$, G. Mirabelli ${ }^{\text {ah }}$, J. Mnich ${ }^{\mathrm{p}}$, G.B. Mohanty ${ }^{\mathrm{j}}$, P. Molnar ${ }^{\text {h }}$, B. Monteleoni ${ }^{\circ}$, T. Moulik ${ }^{j}$, G.S. Muanza w, F. Muheim ${ }^{\mathrm{r}}$, A.J.M. Muijs ${ }^{\text {b }}$, M. Napolitano ${ }^{\text {aa }}$, F. Nessi-Tedaldi ${ }^{\text {at }}$, H. Newman ${ }^{\text {ad }}$, T. Niessen ${ }^{\text {a }}$, A. Nisati ah, H. Nowak as, Y.D. Oh ${ }^{\text {an }}$, G. Organtini ${ }^{\text {ah }}$, R. Ostonen ${ }^{\mathrm{t}}$, C. Palomares ${ }^{x}$, D. Pandoulas ${ }^{a}$, S. Paoletti ${ }^{\text {ah,p }}$, P. Paolucci ${ }^{\text {aa }}$, H.K. Park ${ }^{\text {af }}$, I.H. Park ${ }^{\text {an }}$, G. Pascale ${ }^{\text {ah }}$, G. Passaleva $^{p}$, S. Patricelli ${ }^{\text {aa }}$, T. Paul $^{\text {k }}$, M. Pauluzzi ${ }^{\text {ae }}$, C. Paus ${ }^{\text {p }}$, F. Pauss ${ }^{\text {at }}$, D. Peach ${ }^{\mathrm{p}}$, M. Pedace ${ }^{\text {ah }}$, Y.J. Pei ${ }^{\mathrm{a}}$, S. Pensotti ${ }^{\mathrm{y}}$, D. Perret-Gallix ${ }^{\mathrm{d}}$, B. Petersen ${ }^{\mathrm{ac}}$, D. Piccolo ${ }^{\text {aa }}$, M. Pieri ${ }^{\circ}$, P.A. Piroué ag, E. Pistolesi y , V. Plyaskin ${ }^{\mathrm{z}}$, M. Pohl ${ }^{\text {at }}$, V. Pojidaev ${ }^{\mathrm{z}, \mathrm{o}}$, H. Postema $^{\mathrm{n}}$, J. Pothier ${ }^{\mathrm{p}}$, N. Produit ${ }^{\mathrm{r}}$, D. Prokofiev ai , J. Quartieri aj, G. Rahal-Callot ${ }^{\text {at,p }}$, M.A. Rahaman ${ }^{\mathrm{j}}$, N. Raja ${ }^{j}$, R. Ramelli at, P.G. Rancoita ${ }^{y}$, G. Raven ${ }^{\text {ak }}$, P. Razis ${ }^{a b}$, D. Ren ${ }^{\text {at }}$, M. Rescigno ${ }^{\text {ah }}$, S. Reucroft ${ }^{k}$, T. van Rhee ${ }^{\text {ap }}$, S. Riemann ${ }^{\text {as }}$, K. Riles ${ }^{\text {c, }}$, A. Robohm ${ }^{\text {at }}$, J. Rodin ${ }^{\text {ao }}$, B.P. Roe ${ }^{c}$, L. Romero ${ }^{\mathrm{x}}$, S. Rosier-Lees ${ }^{\mathrm{d}}$, J.A. Rubio ${ }^{\mathrm{p}}$, D. Ruschmeier ${ }^{\text {h }}$, H. Rykaczewski ${ }^{\text {at }}, \mathrm{S}_{\text {. Sarkar }}{ }^{\text {h }}$, J. Salicio ${ }^{\mathrm{p}}$, E. Sanchez ${ }^{\mathrm{p}}$, M.P. Sanders ${ }^{\text {ac }}$, M.E. Sarakinos ${ }^{\text {t }, ~ C . ~ S c h a ̈ f e r ~}{ }^{a}$, V. Schegelsky ${ }^{\text {ai }}$, S. Schmidt-Kaerst a , D. Schmitz a, H. Schopper ${ }^{\text {au }}$, D.J. Schotanus ${ }^{\text {ac }}$, J. Schwenke a G. Schwering a, C. Sciacca ${ }^{\text {aa }}$, D. Sciarrino ${ }^{r}$, A. Seganti i, L. Servoli ae, S. Shevchenko ad, N. Shivarov ${ }^{\text {am }}$, V. Shoutko ${ }^{\text {z }, ~ E . ~ S h u m i l o v ~}{ }^{z}$, A. Shvorob ${ }^{\text {ad }}$, T. Siedenburg ${ }^{\text {a }}$, D. Son ${ }^{\text {an }}$, B. Smith ${ }^{\text {af }}$, P. Spillantini ${ }^{\circ}$, M. Steuer ${ }^{\text {n }}$, 
D.P. Stickland ${ }^{\text {ag }}$, A. Stone ${ }^{\text {f }}$, H. Stone ${ }^{\text {ag }}$, B. Stoyanov ${ }^{\text {am }}$, A. Straessner ${ }^{\text {a }}$,

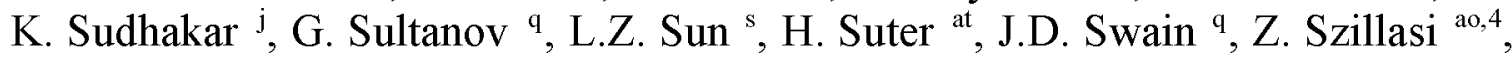
X.W. Tang ${ }^{g}$, L. Tauscher ${ }^{\mathrm{e}}$, L. Taylor ${ }^{\mathrm{k}}$, C. Timmermans ${ }^{\mathrm{ac}}$, Samuel C.C. Ting ${ }^{\mathrm{n}}$, S.M. Ting ${ }^{n}$, S.C. Tonwar ${ }^{\mathrm{j}}$, J. Tóth ${ }^{\mathrm{m}}$, C. Tully ${ }^{\text {ag }}$, K.L. Tung ${ }^{\mathrm{g}}$, Y. Uchida ${ }^{\mathrm{n}}$, J. Ulbricht ${ }^{\text {at }}$, E. Valente ${ }^{\text {ah }}$, G. Vesztergombi ${ }^{\mathrm{m}}$, I. Vetlitsky ${ }^{\mathrm{z}}$, D. Vicinanza ${ }^{\text {aj }}$, G. Viertel ${ }^{\text {at }}$, S. Villa ${ }^{k}$, M. Vivargent ${ }^{\mathrm{d}}$, S. Vlachos ${ }^{\mathrm{e}}$, I. Vodopianov ${ }^{\text {ai }}$, H. Vogel ${ }^{\text {af }}$, H. Vogt ${ }^{\text {as }}$, I. Vorobiev ${ }^{\mathrm{z}}$, A.A. Vorobyov ${ }^{\text {ai }}$, A. Vorvolakos ${ }^{\mathrm{ab}}$, M. Wadhwa ${ }^{\mathrm{e}}$, W. Wallraff a , M. Wang ", X.L. Wang s, Z.M. Wang s, A. Weber ${ }^{\mathrm{a}}$, M. Weber ${ }^{\text {a }}$, P. Wienemann ${ }^{\text {a }}$, H. Wilkens ${ }^{\text {ac }}$, S.X. Wu ${ }^{\text {n }}$, S. Wynhoff ${ }^{\text {a }}$, L. Xia ${ }^{\text {ad }}$, Z.Z. Xu ${ }^{\text {s, B.Z. Yang }}{ }^{\text {s }}$, C.G. Yang ${ }^{\mathrm{g}}$, H.J. Yang ${ }^{\mathrm{g}}$, M. Yang ${ }^{\mathrm{g}}$, J.B. Ye ${ }^{\mathrm{s}}$, S.C. Yeh ${ }^{\text {aw }}$, J.M. You ${ }^{\text {af }}$, An. Zalite ${ }^{\text {ai }}$, Yu. Zalite ${ }^{\text {ai }}$, Z.P. Zhang ${ }^{\text {s }}$, G.Y. Zhu ${ }^{\text {g, }}$ R.Y. Zhu ${ }^{\text {ad }}$, A. Zichichi i,p,q, F. Ziegler ${ }^{\text {as }}$, G. Zilizi ${ }^{\text {ao, } 4}$, M. Zöller ${ }^{\text {a }}$

${ }^{a}$ I. Physikalisches Institut, RWTH, D-52056 Aachen, Germany, and III. Physikalisches Institut, RWTH, D-52056 Aachen, Germany

${ }^{\mathrm{b}}$ National Institute for High Energy Physics, NIKHEF, and University of Amsterdam, NL-1009 DB Amsterdam, The Netherlands ' University of Michigan, Ann Arbor, MI 48109, USA

${ }^{\mathrm{d}}$ Laboratoire d'Annecy-le-Vieux de Physique des Particules, LAPP, IN2P3-CNRS, BP 110, F-74941 Annecy-le-Vieux CEDEX, France

'Insitiute of Physics, University of Basel, CH-4056 Basel, Switzerland

${ }^{\mathrm{f}}$ Louisiana State University, Baton Rouge, LA 70803, USA

${ }^{g}$ Institute of High Energy Physics, IHEP, 100039 Beijing, China ${ }^{6}$

${ }^{\mathrm{h}}$ Humboldt University, D-10099 Berlin, Germany ${ }^{5}$

${ }^{\mathrm{i}}$ University of Bologna and INFN-Sezione di Bologna, I-40126 Bologna, Italy

${ }^{\mathrm{j}}$ Tata Institute of Fundamental Research, Bombay 400005 , India

${ }^{\mathrm{k}}$ Northeastern University, Boston, MA 02115, USA

${ }^{1}$ Institute of Atomic Physics and University of Bucharest, R-76900 Bucharest, Romania

${ }^{\mathrm{m}}$ Central Research Institute for Physics of the Hungarian Academy of Sciences, H-1525 Budapest 114, Hungary ${ }^{7}$

"n Massachusetts Institute of Technology, Cambridge, MA 02139, USA

${ }^{\circ}$ INFN Sezione di Firenze and University of Florence, I-50125 Florence, Italy

${ }^{\mathrm{p}}$ European Laboratory for Particle Physics, CERN, CH-1211 Geneva 23, Switzerland

${ }^{\mathrm{q}}$ World Laboratory, FBLJA Project, $\mathrm{CH}-1211$ Geneva 23, Switzerland

${ }^{\mathrm{r}}$ University of Geneva, $\mathrm{CH}-1211$ Geneva 4, Switzerland

${ }^{s}$ Chinese University of Science and Technology, USTC, Hefei, Anhui 230 029, China ${ }^{6}$

${ }^{\mathrm{t}}$ SEFT, Research Institute for High Energy Physics, P.O. Box 9, SF-00014 Helsinki, Finland

"University of Lausanne, CH-1015 Lausanne, Switzerland

"INFN-Sezione di Lecce and Universitá Degli Studi di Lecce, I-73100 Lecce, Italy

${ }^{\mathrm{w}}$ Institut de Physique Nucléaire de Lyon, IN2P3-CNRS, Université Claude Bernard, F-69622 Villeurbanne, France

${ }^{x}$ Centro de Investigaciones Energéticas, Medioambientales y Tecnologícas, CIEMAT, E-28040 Madrid, Spain ${ }^{8}$

y INFN-Sezione di Milano, I-20133 Milan, Italy

${ }^{\mathrm{z}}$ Institute of Theoretical and Experimental Physics, ITEP, MOscow, Russia

aa INFN-Sezione di Napoli and University of Naples, I-80125 Naples, Italy

${ }^{\mathrm{ab}}$ Department of Natural Sciences, University of Cyprus, Nicosia, Cyprus

${ }^{a}$ University of Nijmegen and NIKHEF, NL-6525 ED Nijmegen, The Netherlands

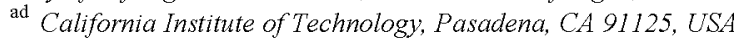

ae INFN-Sezione di Perugia and Universitá Degli Studi di Perugia, I-06100 Perugia, Italy

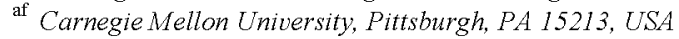

ag Princeton University, Princeton, NJ 08544, USA

ah INFN-Sezione di Roma and University of Rome, 'La Sapienza', I-00185 Rome, Italy

${ }^{\text {ai }}$ Nuclear Physics Institute, St. Petersburg, Russia

aj University and INFN, Salerno, I-84I00 Salerno, Italy

ak University of California, San Diego, CA 92093, USA

${ }^{a}$ Dept. de Fisica de Particulas Elementales, Univ. de Santiago, E-15706 Santiago de Compostela, Spain 


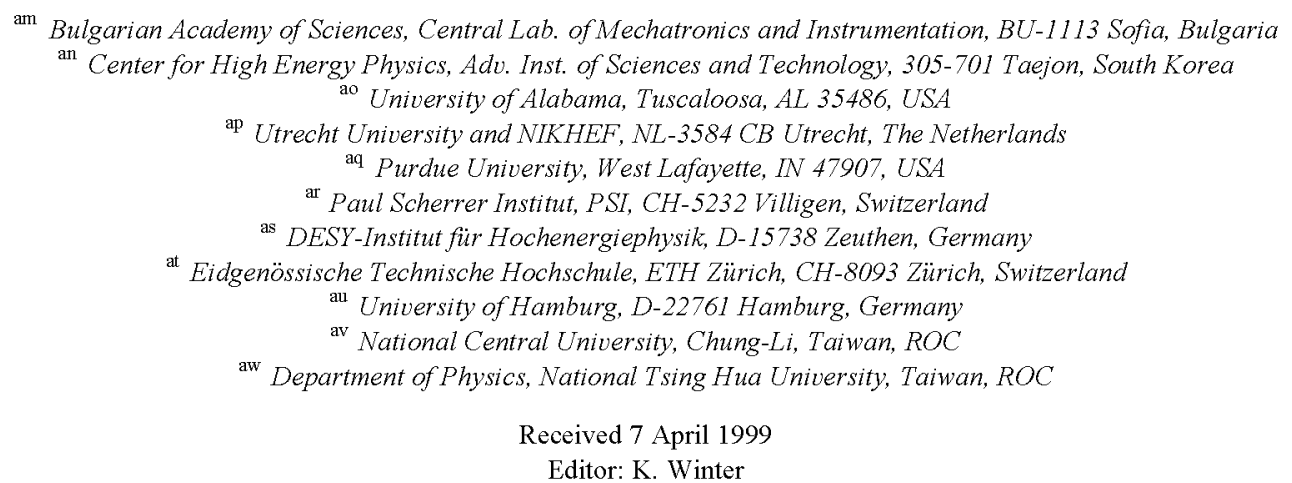

\begin{abstract}
We measure Bose-Einstein correlations between like-sign charged pion pairs in hadronic Z decays with the $\mathrm{L} 3$ detector at LEP. The analysis is performed in three dimensions in the longitudinal center-of-mass system. The pion source is found to be elongated along the thrust axis with a ratio of transverse to longitudinal radius of $0.81 \pm 0.02_{-0.19}^{+0.03}$. (C) 1999 Published by Elsevier Science B.V. All rights reserved.
\end{abstract}

\section{Introduction}

It has long been realized that the shape and size in space-time of a source of pions can be determined, as a consequence of the interference of identical bosons, from the shape and size of the correlation function of two identical pions in energy-momentum space [1-3]. The space-time shape of a source during hadronization is important experimental information on QCD in a sector where perturbative methods are not applicable.

\footnotetext{
${ }^{1}$ Also supported by CONICET and Universidad Nacional de La Plata, CC 67, 1900 La Plata, Argentina.

${ }^{2}$ Also supported by Panjab University, Chandigarh-160014, India.

${ }^{3}$ Supported by Deutscher Akademischer Austauschdienst.

${ }^{4}$ Also supported by the Hungarian OTKA fund under contract numbers T22238 and T026178.

${ }^{5}$ Supported by the German Bundesministerium für Bildung, Wissenschaft, Forschung und Technologie.

${ }^{6}$ Supported by the National Natural Science Foundation of China.

${ }^{7}$ Supported by the Hungarian OTKA fund under contract numbers T019181, F023259 and T024011.

${ }^{8}$ Supported also by the Comisión Interministerial de Ciencia y Tecnología.
}

The form of the correlation function in more than one dimension has been a major subject of theoretical study in recent years [4-10]. In Monte Carlo generators, spherical symmetry is usually assumed [11-15], while elongation can be expected when a string-like shape is maintained $[16,10,9]$. Experimentally, detailed three-dimensional analyses have been done only for heavy ion collisions $[17,18]$ and for hadron-hadron collisions [19]. While the volume of the pion emission region appears to be approximately spherical for heavy ion collisions, a clear elongation is observed in hadron-hadron collisions. Analyses in $\mathrm{e}^{+} \mathrm{e}^{-}$collisions have generally been limited to one dimension [20]. An exception is a preliminary analysis [21], which indicates an elongation in $\mathrm{e}^{+} \mathrm{e}^{-}$collisions.

Recently there has been a revival of interest in Bose-Einstein (BE) correlations of particles produced in the hadronization of energetic quarks, mainly due to its possible impact on the measurements of the $W$ mass [11-16] in the four-jet channel $\mathrm{e}^{+} \mathrm{e}^{-} \rightarrow \mathrm{W}^{+} \mathrm{W}^{-} \rightarrow \mathrm{q} \overline{\mathrm{q}} \mathrm{q} \overline{\mathrm{q}}$. The experimentally observed radius of hadron emission is of order $1 \mathrm{fm}$, about an order of magnitude larger than the distance between the $\mathrm{W}^{+} \mathrm{W}^{-}$decay vertices at present ener- 
gies. The actual overlap in $\mathrm{W}^{+} \mathrm{W}^{-} \rightarrow \mathrm{q} \overline{\mathrm{q}} \mathrm{q} \overline{\mathrm{q}}$ decays is of course determined by the (possibly non-spherical) configuration of the decay partons of the W's in all space-time dimensions during hadronization. $A$ better understanding of BE correlations in $\mathrm{e}^{+} \mathrm{e}^{-} \rightarrow Z \rightarrow$ $\mathrm{q} \overline{\mathrm{q}}$, using high statistics LEP data, will contribute to understanding the $\mathrm{BE}$ effect in $\mathrm{W}^{+} \mathrm{W}^{-}$.

In this letter, two-particle correlations of likecharged pions are studied in hadronic Z-decay as a function of three components of the four-momentum difference $Q$. Effects due to hard gluon radiation, Z-decay into heavy quarks or the dependence on the transverse mass of the particles are not considered.

\section{Data}

\subsection{Charged-particle and event selection}

The data used in the analysis were collected by the $\mathrm{L} 3$ detector [22] in 1994 at a center-of-mass energy of $\sqrt{s} \simeq 91.2 \mathrm{GeV}$. The data selection uses information on charged particles from the Time Expansion Chamber (TEC) and the Silicon Microvertex Detector (SMD) [22].

To obtain a data sample of hadronic $Z$ decays, we perform an event selection using charged tracks. The charged tracks are required to have at least 40 (of 62 possible) hits in TEC, and the number of wires between the first and last hit is required to be at least 50. The distance of closest approach (projected onto the transverse plane) of a track to the nominal interaction vertex is required to be less than $5 \mathrm{~mm}$. The transverse momentum of a track must be greater than $100 \mathrm{MeV}$.

In order to reduce background arising from beamgas and beam-wall interactions as well as from leptonic events and from two-photon interactions, we use the following criteria

$$
\begin{aligned}
& \frac{\sum_{i}\left|\boldsymbol{p}_{i}\right|}{\sqrt{s}}>0.15, \quad \frac{\left|\sum_{i} p_{\| i}\right|}{\sum_{i}\left|\boldsymbol{p}_{i}\right|}<0.75, \\
& \frac{\left|\sum_{i} \boldsymbol{p}_{\perp i}\right|}{\sum_{i}\left|\boldsymbol{p}_{i}\right|}<0.75, \quad N_{\mathrm{ch}}>4,
\end{aligned}
$$

where $\boldsymbol{p}_{i}$ is the momentum of particle $i$, with components $p_{\| i}$ parallel to the beam direction and $\boldsymbol{p}_{\perp i}$ in the plane perpendicular to the beam direction, and where the sums run over all the tracks of the event. $N_{\text {ch }}$ is the number of charged-particle tracks. A small remaining background of $\tau^{+} \tau^{-}$events in which both $\tau$ particles decay into more than one charged particle is removed by requiring the second largest angle $\varphi_{2}$ between any two neighboring tracks in the $R-\varphi$ plane to be less than $170^{\circ}$. After this cut the $\tau^{+} \tau^{-}$ contamination is approximately $8 \cdot 10^{-5}$.

To make sure that events lie within the full acceptance of the TEC and SMD, we require $\left|\cos \theta_{\mathrm{thr}}\right|<0.7$, where $\theta_{\mathrm{thr}}$ is the polar angle of the thrust axis determined from charged tracks only.

A total of about one million events satisfy the selection criteria.

For the computation of the four-momentum difference $Q$, the resolution of the angle between pairs of tracks is of crucial importance, especially for small $Q$-values. For this reason we impose an additional cut, requiring an unambiguous polar angle measurement. This ensures good resolution of variables, such as $Q$, which depend on two tracks. Together with the previous cuts about $40 \%$ of the
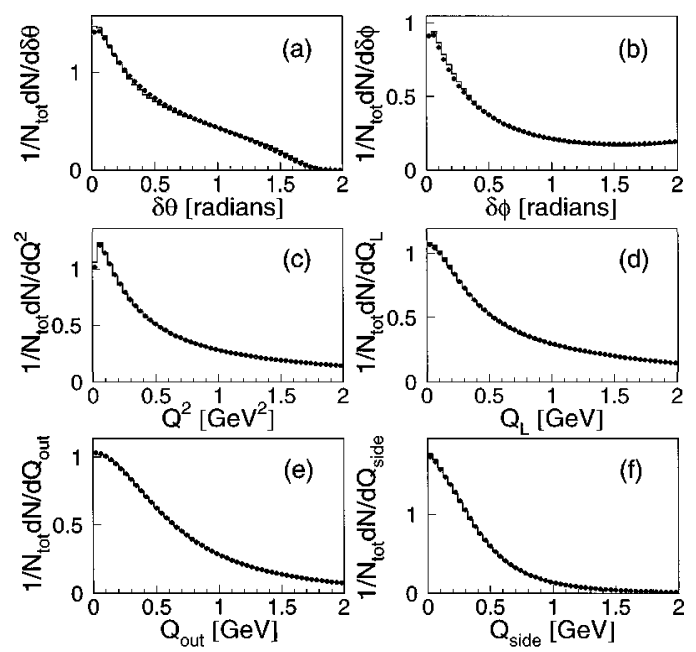

Fig. 1. Distributions of (a) the difference in polar angle of pairs of tracks, $\delta \theta$, (b) the difference in azimuthal angle of pairs of tracks, $\delta \phi$, (c) the four-momentum difference squared, $Q^{2}$, and the (d) longitudinal, (e) out, and (f) side components of the four-momentum difference $Q$, for data (points) compared to the predictions of JETSET with BE after detector simulation (histogram). 
tracks are rejected. The final data sample consists of approximately 36 million like-sign track pairs.

With this selection, good agreement is obtained between data and simulation for the distributions of the differences between pairs of tracks of the azimuthal and polar angles with respect to the beam and for the distributions of $Q^{2}$ and the components of $Q$ used in this analysis. This is shown in Fig. 1, where the data are compared to events generated with JETSET [23] (including BE effects) which have been passed through the $\mathrm{L} 3$ detector simulation program [24], reconstructed, and subjected to track and event selection in the same way as the data. Similar comparisons using events generated by JETSET without BE effects or by HERWIG [25] also show good agreement except at small values of the variables, where the Bose-Einstein effect is important.

\section{Analysis}

\subsection{Bose-Einstein correlation function}

The two-particle correlation function of two particles with four-momenta $p_{1}$ and $p_{2}$ is given by the ratio of the two-particle number density, $\rho_{2}\left(p_{1}, p_{2}\right)$, to the product of the two single-particle number densities, $\rho_{1}\left(p_{1}\right) \rho_{1}\left(p_{2}\right)$. Since we are here interested only in the correlation $R_{2}$ due to Bose-Einstein interference, the product of single-particle densities is replaced by $\rho_{0}\left(p_{1}, p_{2}\right)$, the two-particle density that would occur in the absence of Bose-Einstein correlations:

$R_{2}\left(p_{1}, p_{2}\right)=\frac{\rho_{2}\left(p_{1}, p_{2}\right)}{\rho_{0}\left(p_{1}, p_{2}\right)}$.

$R_{2}-1$ is related to the space-time particle density through a Fourier transform [1,2].

Since the mass of the two identical particles of the pair is fixed to the pion mass, the correlation function is defined in six-dimensional momentum space. Since Bose-Einstein correlations can be large only at small four-momentum difference $Q=\sqrt{-\left(p_{1}-p_{2}\right)^{2}}$, they are often parametrized in this one-dimensional distance measure. There is no reason, however, to expect the hadron source to be spherically symmetric in jet fragmentation. This is the reason for performing a three-dimensional analysis.

\subsection{Longitudinal center-of-mass system}

In our analysis we use the longitudinal center-ofmass system (LCMS) [7]. This is defined for each pair of particles as the system, resulting from a boost along the thrust axis, in which the sum of the momenta of the pair is perpendicular to the thrust axis. In this system, we can resolve the threemomentum difference of the pair of particles into a longitudinal component $Q_{\mathrm{L}}$ parallel to the thrust axis, $Q_{\text {out }}$ along the sum of the particles' momenta (see Fig. 2) and $Q_{\text {side }}$ perpendicular to both $Q_{\mathrm{L}}$ and $Q_{\text {out }}$. Then, the invariant four-momentum difference can be written as [7]

$$
\begin{aligned}
Q^{2} & =Q_{\mathrm{L}}^{2}+Q_{\text {side }}^{2}+Q_{\text {out }}^{2}-(\Delta E)^{2} \\
& =Q_{\mathrm{L}}^{2}+Q_{\text {side }}^{2}+Q_{\text {out }}^{2}\left(1-\beta^{2}\right),
\end{aligned}
$$

where

$$
\beta \equiv \frac{p_{\text {out } 1}+p_{\text {out } 2}}{E_{1}+E_{2}},
$$

with $p_{\text {out } i}$ and $E_{i}(i=1,2)$ the out-component of the momentum and the energy, respectively, of particle $i$ in the LCMS. The energy difference $\Delta E$ and therefore the difference in emission time of the two particles couples only to the component $Q_{\text {out }}$. Consequently, $Q_{\mathrm{L}}$ and $Q_{\text {side }}$ reflect only spatial dimensions of the source, whereas $Q_{\text {out }}$ reflects a mixture of spatial and temporal dimensions. The correlation

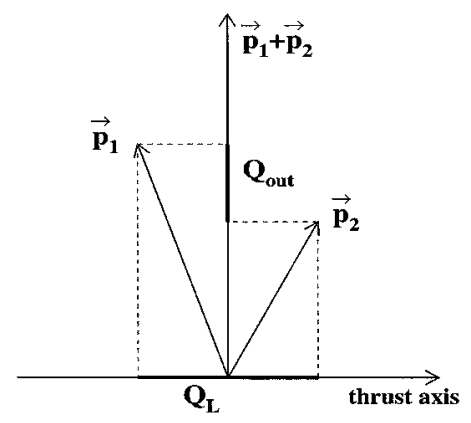

Fig. 2. The longitudinal center of mass frame (LCMS) showing the projection of $Q$ on the $\left(Q_{\mathrm{L}}-Q_{\text {out }}\right)$ plane. $Q_{\text {side }}$ is the projection of $Q$ on the axis perpendicular to this plane. 
function is then parametrized in terms of $Q=$ $\left(Q_{\mathrm{L}}, Q_{\text {side }}, Q_{\text {out }}\right)$ :

$R_{2}(\boldsymbol{Q})=\frac{\rho_{2}(\boldsymbol{Q})}{\rho_{0}(\boldsymbol{Q})}$.

\subsection{Determination of the correlation function $R_{2}$}

After determining $\rho_{2}(\boldsymbol{Q})$, there are three steps in obtaining $R_{2}$. The first step is to create a 'reference sample', which is done by event mixing, and from it to determine $\rho_{0}(\boldsymbol{Q})$. Then two corrections must be determined, one for non-BE correlations lost in the event mixing and one for detector effects.

The reference sample, from which $\rho_{0}$ is determined, is formed by mixing particles from different data events in the following way. First, events are rotated to a system with the $z$-axis along the thrust axis and are stored in a 'pool'. Then, events are randomly selected from this pool and their tracks replaced by tracks of the same charge from other events in the pool of approximately the same multiplicity under the condition that no track originates from the same event. Finally, $Q_{\mathrm{L}}, Q_{\text {out }}$ and $Q_{\text {side }}$ are calculated for each pair. Used events are removed from the pool, thus preventing any regularities in the reference sample. From this mixed sample we obtain the particle density $\rho_{\text {mix }}(\boldsymbol{Q})$.

Since this mixing procedure removes correlations other than just those of Bose-Einstein, e.g., those from energy-momentum conservation and from resonances, $\rho_{\mathrm{mix}}$ is then corrected for this loss by a factor $C_{\mathrm{mix}}$, which is estimated by Monte Carlo (MC) using a generator with no Bose-Einstein (BE) effects (JETSET or HERWIG). Thus, in the absence of Bose-Einstein correlations, the corrected two-particle density is given by

$$
\begin{aligned}
& \rho_{0}(\boldsymbol{Q})=\rho_{\mathrm{mix}}(\boldsymbol{Q}) \cdot C_{\mathrm{mix}}(\boldsymbol{Q}) \\
& \text { where } \quad C_{\mathrm{mix}}(\boldsymbol{Q})=\left[\frac{\rho_{2}(\boldsymbol{Q})}{\rho_{\mathrm{mix}}(\boldsymbol{Q})}\right]_{\mathrm{MC}, \mathrm{noBE}} .
\end{aligned}
$$

The ratio $\left(\rho_{2} / \rho_{\text {mix }}\right)$ must further be corrected for detector resolution, acceptance and efficiency and for particle misidentification. For this we use a multiplicative factor derived from Monte Carlo studies. Since the $\mathrm{L} 3$ detector does not identify the hadrons, this factor, $C_{\text {det }}$, is given by the ratio of the two-pion correlation function found from $\mathrm{MC}$ events at generator level to the two-particle correlation function found using all particles after full detector simulation, reconstruction and selection:

$$
C_{\mathrm{det}}(\boldsymbol{Q})=\left(\frac{\rho_{2}(\boldsymbol{Q})}{\rho_{\mathrm{mix}}(\boldsymbol{Q})}\right)_{\mathrm{gen}, \text { pions }} /\left(\frac{\rho_{2}(\boldsymbol{Q})}{\rho_{\mathrm{mix}}(\boldsymbol{Q})}\right)_{\mathrm{det}, \text { all }} .
$$

Taking all charged particles, instead of only pions, in the generator level MC, leads to consistent results. Combining this correction factor with (5) and (6) results in

$R_{2}(Q)=\frac{\rho_{2}(\boldsymbol{Q})}{\rho_{\mathrm{mix}}(\boldsymbol{Q})} \cdot \frac{1}{C_{\mathrm{mix}}(\boldsymbol{Q})} \cdot C_{\mathrm{det}}(\boldsymbol{Q})$.

The analysis is done in three-dimensional bins of $\boldsymbol{Q}$. In terms of numbers $N_{k l m}$ of like-sign particle pairs in the three-dimensional bin $k, l, m$ of $Q_{\mathrm{L}}, Q_{\text {out }}$ and $Q_{\text {side }}$, Eq. (8) becomes

$$
\begin{aligned}
R_{2 k l m}= & {\left[\frac{N_{k l m}}{N_{k l m}^{\mathrm{mix}}}\right]_{\mathrm{data}}\left[\frac{N_{k l m}^{\mathrm{mix}}}{N_{k l m}}\right]_{\mathrm{MC}, \mathrm{noBE}} } \\
& \cdot\left[\frac{N_{k l m}^{\mathrm{gen}}}{N_{k l m}^{\mathrm{gen}, \mathrm{mix}}} \cdot \frac{N_{k l m}^{\mathrm{det}, \mathrm{mix}}}{N_{k l m}^{\mathrm{det}}}\right]_{\mathrm{MC}} .
\end{aligned}
$$

Each $N_{k l m}$ is normalized to the total number of pairs in the corresponding sample.

The resolution in the variables $Q_{i}$ is estimated using Monte Carlo events to be $0.02-0.05 \mathrm{GeV}$ for $Q_{i}<0.2 \mathrm{GeV}$. Given the available statistics, we choose a bin size of $0.08 \mathrm{GeV}$.

In our analysis, we use JETSET without BE and HERWIG (not having BE) to determine the mixing correction factor $C_{\text {mix }}$ and JETSET (with and without BE) as well as HERWIG to determine the detector correction factor $C_{\text {det }}$. Together with a variation of the mixing technique, the selection criteria and the fit range, these six Monte Carlo combinations will serve to estimate systematic errors.

\subsection{Parametrization of $R_{2}$}

Assuming a Gaussian (azimuthally, but not necessarily spherically, symmetric) shape of the source, 
the following three-dimensional parametrization has been proposed $[5,6,26]$ :

$$
\begin{aligned}
R_{2}\left(Q_{\mathrm{L}}, Q_{\text {out }}, Q_{\text {side }}\right) & \\
= & \gamma\left(1+\delta Q_{\mathrm{L}}+\varepsilon Q_{\text {out }}+\xi Q_{\text {side }}\right) \\
& \cdot\left[1+\lambda \exp \left(-R_{\mathrm{L}}^{2} Q_{\mathrm{L}}^{2}-R_{\text {out }}^{2} Q_{\text {out }}^{2}-R_{\text {side }}^{2} Q_{\text {side }}^{2}\right.\right. \\
& \left.\left.+2 \rho_{\mathrm{L}, \text { out }} R_{\mathrm{L}} R_{\text {out }} Q_{\mathrm{L}} Q_{\text {out }}\right)\right]
\end{aligned}
$$

where the factor $\left(1+\delta Q_{\mathrm{L}}+\varepsilon Q_{\text {out }}+\xi Q_{\text {side }}\right)$ takes into account possible long-range momentum correlations in the form of a slow rise, $\gamma$ is a normalization factor close to unity and the term between square brackets is the two-particle Bose-Einstein correlation function associated with a Gaussian shape of the source.

By fitting the correlation function with this parametrization, one can extract the incoherence factor $\lambda$, which measures the strength of the correlation, and the 'radii' $R_{i}$ ( $i=\mathrm{L}$, out and side) defined as $1 / \sqrt{2} \sigma_{i}$, with $\sigma_{i}^{2}$ the variances of a multi-dimensional Gaussian distribution of the source in configuration space. $\rho_{\mathrm{L}, \text { out }}$ is the correlation between the longitudinal and out components of this Gaussian. In the LCMS, the duration of particle emission only couples to the out-direction and only enters in the parameters $R_{\text {out }}$ and $\rho_{\mathrm{L} \text {,out }}$. Hence, $R_{\text {side }}$ can be interpreted as the transverse component of the geometric radius. The parametrization, Eq. (10), assumes azimuthal symmetry of the source, which means that the two-particle Bose-Einstein correlation function associated with the Gaussian shape of the source, is invariant under the transformation $Q_{\text {side }} \rightarrow$ $-Q_{\text {side }}$. Consequently, the only possible off-diagonal term is the $Q_{\mathrm{L}} Q_{\text {out }}$ term.

We first checked the method using Monte Carlo events at detector level, which were generated by JETSET without BE, instead of data. The correction factor $C_{\text {mix }}$ was determined using JETSET without $\mathrm{BE}$ and using HERWIG, while $C_{\text {det }}$ was determined using JETSET with BE and using HERWIG, giving four different combinations of correction factors. Also we used events generated by HERWIG as data, with the same two MC determinations of $C_{\text {mix }}$ but with JETSET with $\mathrm{BE}$ and JETSET without $\mathrm{BE}$ for $C_{\mathrm{det}}$. In all eight cases fits gave results consistent with $\lambda=0\left(\chi^{2} / \mathrm{NDF} \simeq 1\right.$ for $\lambda=0$ ), as expected in the absence of Bose-Einstein correlations.

\section{Results}

The results of a three-dimensional fit, in the range $Q_{i}<1.04 \mathrm{GeV}$, of the Bose-Einstein correlation function $R_{2}$ with the parametrization of Eq. (10) are presented in Table 1 . The off-diagonal term turns out to be zero within errors $\left(\rho_{\mathrm{L}, \text { out }}=-0.008 \pm 0.057\right)$ and the results given in the table correspond to a fit with this term fixed to zero. The values are obtained using JETSET without $\mathrm{BE}$ for the mixing correction and JETSET with BE for the detector correction, since the latter model is found to be the most successful in reproducing our data in the relevant variables (Fig. 1) and since this choice gives the lowest $\chi^{2}$ in the fits.

To estimate the systematic errors on the fit parameters, we examined four different sources, repeating the full analysis in each case. First, we looked at the fit results obtained with the six possible combinations of mixing and detector Monte Carlo corrections. The systematic error from this source is taken as the RMS of these six values. Secondly, the influence of a different mixing sample was studied by removing the conditions that tracks are replaced by tracks with the same charge and coming from events

Table 1

Values of the fit parameters for the three-dimensional analyses of L3 data. The first error is statistical, the second systematic

\begin{tabular}{ccc}
\hline parameter & Gaussian & Edgeworth \\
\hline$\lambda$ & $0.41 \pm 0.01_{-0.19}^{+0.02}$ & $0.54 \pm 0.02_{-0.26}^{+0.04}$ \\
$R_{\mathrm{L}}(\mathrm{fm})$ & $0.74 \pm 0.02_{-0.03}^{+0.04}$ & $0.69 \pm 0.02_{-0.03}^{+0.04}$ \\
$R_{\text {out }}(\mathrm{fm})$ & $0.53 \pm 0.02_{-0.06}^{+0.05}$ & $0.44 \pm 0.02_{-0.06}^{+0.05}$ \\
$R_{\text {side }}(\mathrm{fm})$ & $0.59 \pm 0.01_{-0.13}^{+0.03}$ & $0.56 \pm 0.02_{-0.12}^{+0.03}$ \\
$R_{\text {out }} / R_{\mathrm{L}}$ & $0.71 \pm 0.02_{-0.08}^{+0.05}$ & $0.65 \pm 0.03_{-0.09}^{+0.06}$ \\
$R_{\text {side }} / R_{\mathrm{L}}$ & $0.80 \pm 0.02_{-0.18}^{+0.03}$ & $0.81 \pm 0.02_{-0.19}^{+0.03}$ \\
& & $0.5 \pm 0.1_{-0.2}^{+0.1}$ \\
$\kappa_{\mathrm{L}}$ & - & $0.8 \pm 0.1 \pm 0.3$ \\
$\kappa_{\text {out }}$ & - & $0.1 \pm 0.1 \pm 0.3$ \\
$\kappa_{\text {side }}$ & - & $0.036 \pm 0.007_{-0.023}^{+0.012}$ \\
$\delta$ & $0.025 \pm 0.005_{-0.015}^{+0.014}$ & $0.011 \pm 0.005_{-0.037}^{+0.037}$ \\
$\epsilon$ & $0.005 \pm 0.005_{-0.012}^{+0.034}$ & $-0.022 \pm 0.006_{-0.025}^{+0.020}$ \\
$\xi$ & $-0.035 \pm 0.005_{-0.024}^{+0.031}$ & $2220 / 2186$ \\
$\chi^{2} / \mathrm{NDF}$ & $2314 / 2189$ & 30 \\
C.L. $(\%)$ & 3.1 & \\
\hline
\end{tabular}


with approximately the same multiplicity. For each of the six Monte Carlo combinations the difference in the results between the two mixing methods was taken as an estimate of the systematic error from this source and the square root of the mean of the squares of these differences taken as the systematic error from this source. In the same way systematic errors related to track/event selection and to the choice of fit range were evaluated. The analysis was repeated with stronger and weaker selection criteria, resulting in approximately $11 \%$ fewer/more events and $6 \%$ fewer/more tracks. The upper limit of the fit range was varied by $\pm 0.16 \mathrm{GeV}$ in all $Q_{i}$. As a total systematic error (second error in the table) we add the four errors from these sources in quadrature.

For $R_{\mathrm{L}}$ the errors from all the sources are approximately equal. However, the systematic error on $\lambda$ and the transverse radii is dominated by the contribution from the six possible MC combinations to determine $C_{\mathrm{det}} \cdot C_{\mathrm{mix}}$. Of the two, $C_{\mathrm{det}}$ shows the larger generator dependence and accounts for most of the systematic error. It is approximately unity for most values of $Q$, but increases to about 1.1 near $Q=0$ in the case of JETSET with BE and decreases to about 0.95 for JETSET without $\mathrm{BE}$ and to about 0.9 for HERWIG. We understand this difference in behavior between JETSET with BE and the two Monte Carlos without BE as follows. Since $Q$ depends both on the energies of the particles as well as on the angle between them, small $Q$ can be due to either small angle or low energies. In a Monte Carlo with BE the fraction of pairs at small $Q$ with small angle will be larger than in the other Monte Carlo models. This will lead to lower detection efficiency and hence to larger corrections. This part of the error is also responsible for the asymmetry in the errors since our best choice of MC combination does not coincide with the average. In particular, all of the other combinations result in smaller values of $R_{\text {side }} / R_{\mathrm{L}}$.

To study the behavior of the components of $Q$, projections of $R_{2}$, Eq. (9), onto the three axes are shown in Fig. 3, using the regions $Q_{i}<240 \mathrm{MeV}$ (i.e., the first three bins) of the non-projected components. The dashed curves correspond to the fit results described above. Similarly, a projection of $R_{2}$ onto the $Q_{\text {side }}-Q_{\mathrm{L}}$ plane is shown in Fig. 4. From Table 1 we find that $R_{\mathrm{L}}$ is larger than both $R_{\text {out }}$ and $R_{\text {side }}$. The ratios $R_{\text {side }} / R_{\mathrm{L}}$ and $R_{\text {out }} / R_{\mathrm{L}}$ are 5 standard
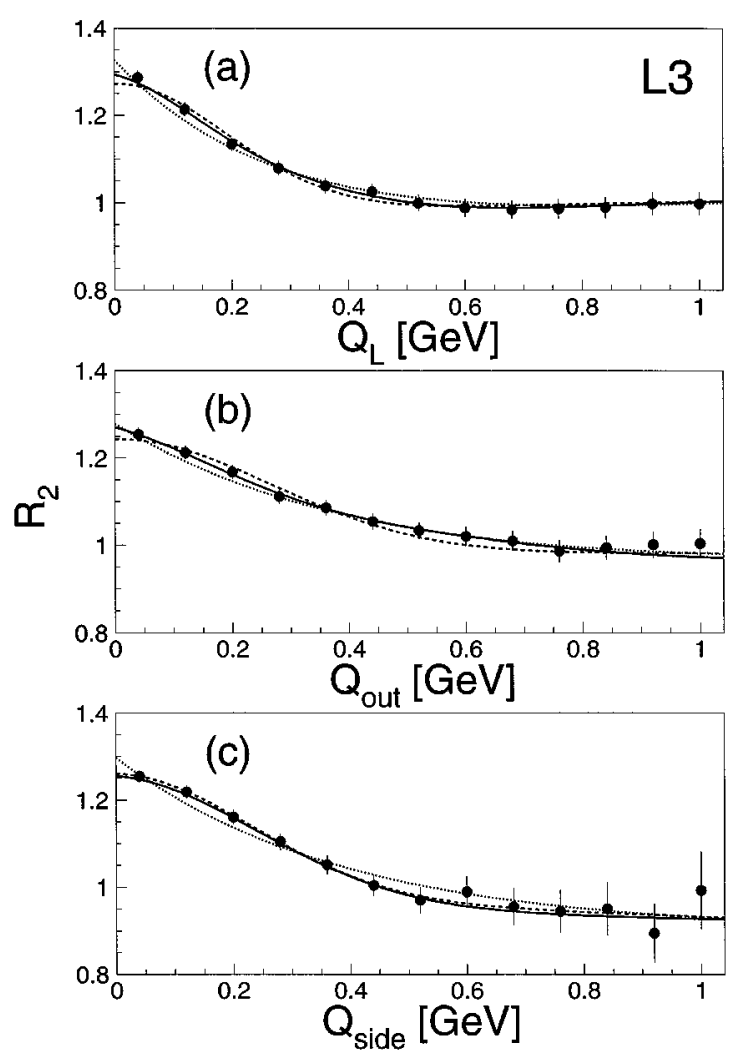

Fig. 3. Projections of $R_{2}$ onto the three axes $Q_{\mathrm{L}}, Q_{\text {out }}$ and $Q_{\text {side }}$ using the regions up to $240 \mathrm{MeV}$ of the non-projected components. The mixing correction is determined using JETSET without $\mathrm{BE}$ and the detector correction using JETSET with BE. The full lines correspond to projections of the fit with the lowest-order Edgeworth expansion, the dashed lines to those of the Gaussian fit and the dotted lines to the exponential fit.

deviations less than unity. Thus, the pion source is elongated along the thrust axis.

From the value of $\chi^{2}$ (see Table 1) it appears that the shape of the correlation function deviates from a Gaussian. An exponential has often been suggested as an alternative to the Gaussian. Accordingly, the fits were repeated with an exponential, $\exp \left(-\sum_{i} R_{i} Q_{i}\right)$, replacing the Gaussian in Eq. (10) with $\rho_{\mathrm{L}, \text { out }}=0$ (dotted lines in Fig. 3). Although the overall $\chi^{2}$ increases by typically $2-4 \%$, depending on the correction combination used, the fits confirm the elongation observed from the Gaussian fit.

A general approach to study deviations from the Gaussian [27], is to use an expansion, due to Edgeworth [28], in terms of derivatives of the Gaussian, 


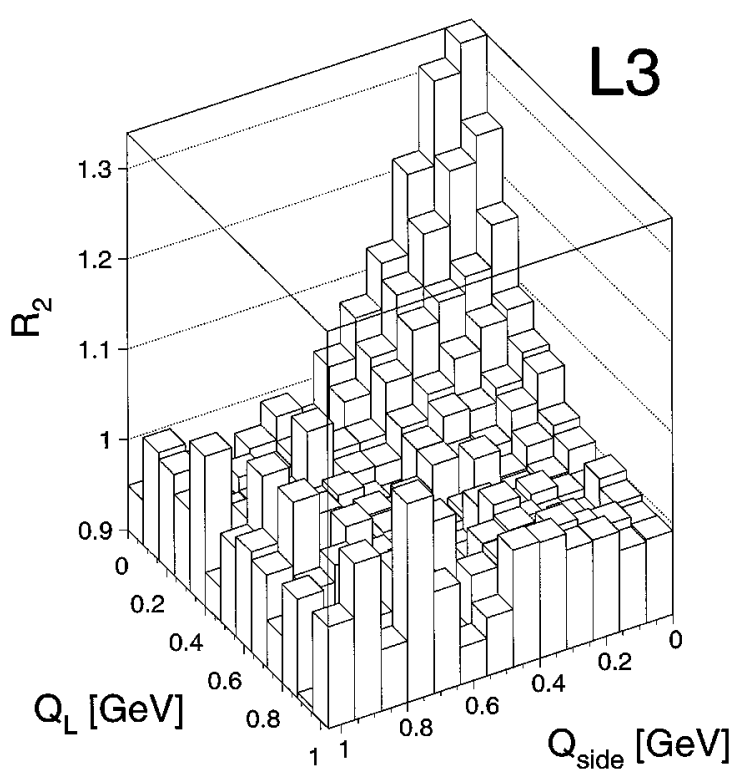

Fig. 4. Projection of $R_{2}$ onto the $Q_{\text {side }}-Q_{\mathrm{L}}$ plane using the regions up to $240 \mathrm{MeV}$ of $Q_{\text {out }}$. The mixing correction is determined using JETSET without $\mathrm{BE}$ and the detector correction using JETSET with $\mathrm{BE}$.

which are related to Hermite polynomials. Taking only the lowest-order non-Gaussian term into account, results in

$$
\begin{aligned}
R_{2}\left(Q_{\mathrm{L}}, Q_{\text {out }}, Q_{\text {side }}\right) \\
=\gamma\left(1+\delta Q_{\mathrm{L}}+\varepsilon Q_{\text {out }}+\xi Q_{\text {side }}\right) \\
\cdot\left\{1+\lambda \exp \left(-R_{\mathrm{L}}^{2} Q_{\mathrm{L}}^{2}-R_{\text {out }}^{2} Q_{\text {out }}^{2}-R_{\text {side }}^{2} Q_{\text {side }}^{2}\right)\right. \\
\cdot\left[1+\frac{\kappa_{\mathrm{L}}}{3 !} H_{3}\left(R_{\mathrm{L}} Q_{\mathrm{L}}\right)\right] \\
\cdot\left[1+\frac{\kappa_{\text {out }}}{3 !} H_{3}\left(R_{\text {out }} Q_{\text {out }}\right)\right] \\
\left.\cdot\left[1+\frac{\kappa_{\text {side }}}{3 !} H_{3}\left(R_{\text {side }} Q_{\text {side }}\right)\right]\right\}
\end{aligned}
$$

where $\kappa_{i}(i=\mathrm{L}$, out,side $)$ is the third-order cumulant moment in the corresponding direction and $H_{3}\left(R_{i} Q_{i}\right) \equiv\left(\sqrt{2} R_{i} Q_{i}\right)^{3}-3 \sqrt{2} R_{i} Q_{i}$ is the third-order Hermite polynomial. Note that the second-order cumulant corresponds to the radius $R_{i}$. The results of the fit with the Edgeworth expansion are shown in Table 1 and Fig. 3 (full lines). The value of $\chi^{2} / \mathrm{NDF}$ and the confidence level indicate a better fit than the Gaussian one. The non-zero values of the $\kappa$ parameters indicate the deviation from a Gaussian. $\lambda$ is larger than the corresponding Gaussian $\lambda$. The values of the radii confirm the elongation observed from the Gaussian fit.

For comparison we have also performed fits on a JETSET generator sample taking $R_{2}$ as

$R_{2, \mathrm{JETSET}}=\frac{\left(N / N^{\mathrm{mix}}\right)_{\mathrm{JETSET} \text { with BE }}}{\left(N / N^{\text {mix }}\right)_{\mathrm{JETSET} \text { no BE }}}$.

The resulting values of $R_{\text {side }} / R_{\mathrm{L}}$ and $R_{\text {out }} / R_{\mathrm{L}}$ are $1.08 \pm 0.03$ and $0.79 \pm 0.02$, respectively. This value of $R_{\text {side }} / R_{\mathrm{L}}$ is larger than in the data (see Table 1 ). Thus, the standard Bose-Einstein implementation of JETSET $^{9}$ fails to reproduce the experimentally observed elongation. It is worth noting that even though there is no explicit spatial asymmetry in the JETSET treatment of $\mathrm{BE}$, neither $R_{\text {side }}$ nor $R_{\text {out }}$ is found to be equal to $R_{\mathrm{L}}$.

Another Monte Carlo generator with Bose-Einstein simulation is VNI [29], which in its present form has been found to predict $R_{\text {side }} \approx R_{\mathrm{L}}$ [15], in contradiction to our results.

Since the difference between the two transverse components of $R$ is small compared to that between the longitudinal component and either of the transverse components, we check the results of the threedimensional analysis by a two-dimensional analysis in $R_{\mathrm{L}}$ and $R_{\mathrm{T}}$, where we can use intervals of 40 instead of $80 \mathrm{MeV}$. The out and side terms in the exponential of Eq. (10) are replaced by $R_{\mathrm{T}}^{2} Q_{\mathrm{T}}^{2}$, with $Q_{\mathrm{T}}^{2}=Q_{\text {out }}^{2}+Q_{\text {side. }}^{2}$. The two-dimensional fits (both Gaussian and Edgeworth) result in values of $\lambda$ consistent with those from the three-dimensional fits and with values of $R_{\mathrm{T}} / R_{\mathrm{L}}$ lying between the values of $R_{\text {out }} / R_{\mathrm{L}}$ and $R_{\text {side }} / R_{\mathrm{L}}$, confirming the elongation observed in the three-dimensional fits. As in the three-dimensional fits, the confidence level of the Gaussian fit is poor, that of the exponential fit poorer, and that of the Edgeworth fit acceptable. These statements are also true for analyses of JETSET.

\footnotetext{
${ }^{9}$ The Bose-Einstein simulation is done by the subroutine LUBOEI, with the $\mathrm{L} 3$ default values $\operatorname{PARJ}(92)=1.5$ and $\operatorname{PARJ}(93)=$ $0.33 \mathrm{GeV}$.
} 


\section{Summary}

A sample of 1 million events of the electronpositron annihilation process $\mathrm{e}^{+} \mathrm{e}^{-} \rightarrow Z \rightarrow$ hadrons at LEP, has been used to study two-particle Bose-Einstein correlations of like-charged pions. The analysis was performed in three dimensions, as well as in two dimensions, in the longitudinal center-of-mass system. Using the Gaussian parametrization of Eq. (10) we extracted the incoherence factor, $\lambda$, and the radii, $R_{\mathrm{L}}, R_{\text {out }}$ and $R_{\text {side }}$. A better fit was obtained using the Edgeworth expansion (11) of the Gaussian parametrization. The transverse radius is found to be significantly smaller than the longitudinal radius:

$$
\frac{R_{\text {side }}}{R_{\mathrm{L}}}=0.81 \pm 0.02_{-0.19}^{+0.03} \text {. }
$$

Our measurement implies that models based on the assumption of a spherical source are too simple.

\section{Acknowledgements}

We wish to express our gratitude to the CERN accelerator divisions for the excellent performance of LEP. We acknowledge the effort of the engineers and technicians who have contributed to the construction and maintenance of the experiment. Clarifying discussions with T. Csörgő, O. Smirnova, Š. Todorova-Nová are gratefully acknowledged.

\section{References}

[1] G. Goldhaber, S. Goldhaber, W. Lee, A. Pais, Phys. Rev. 120 (1960) 300.

[2] D.H. Boal, C.K. Gelbke, B.K. Jennings, Rev. Mod. Phys. 62 (1990) 553

[3] G. Baym, Acta Phys. Polonica B 29 (1998) 1839.

[4] F. Yano, S. Koonin, Phys. Lett. B 78 (1978) 556; M. Podgoretskii, Sov. J. Nucl. Phys. 37 (1983) 272.

[5] G. Bertsch, M. Gong, M. Tohyama, Phys. Rev. C 37 (1988) 1896.

[6] S. Pratt, T. Csörgő, J. Zimanyi, Phys. Rev. C 42 (1990) 2646.

[7] T. Csörgö, S. Pratt, in: Csörgö et al. (Eds.), Proc. Workshop on Relativistic Heavy-Ion Physics, KFKI-1991-28/A, Budapest, 1991, p. 75 .
[8] U. Heinz, Phys. Lett. B 382 (1996) 181

[9] T. Csörgö, B. Lörstad, in: D. Bruncko et al. (Eds.), Proc. XXVth Int. Symp. on Multiparticle Dynamics, World Scientific, Singapore, 1997, p. 66.

[10] B. Andersson, M. Ringnér, Phys. Lett. B 421 (1998) 283; Nucl. Phys. B 513 (1998) 627.

[11] L. Lönnblad, T. Sjöstrand, Phys. Lett. B 351 (1995) 293; Eur. Phys. J. C 2 (1998) 165.

[12] S. Jadach, K. Zalewski, Acta Phys. Polonica B 28 (1997) 1363.

[13] K. Fiałkowski, R. Wit, Z. Phys. C 74 (1997) 145.

[14] V. Kartvelishvili, R. Kvatadze, R. Møller, Phys. Lett. B 408 (1997) 331.

[15] K. Geiger et al., Bose-Einstein correlations in a phase-space approach to $\mathrm{e}^{+} \mathrm{e}^{-}$annihilation into hadrons, CERN-TH/98345

[16] Ц̆. Todorova-Nová, J. Rameš, Simulation of Bose-Einstein effect using space-time aspects of Lund string fragmentation model, Strasbourg preprint IReS97-29.

[17] H. Becker et al., NA44 Collaboration, Z. Phys. C 64 (1994) 209; Phys. Rev. Lett. 74 (1995) 3340.

[18] D. Ferenc et al., NA35 Collaboration, Nucl. Phys. A 544 (1992) 531c; Th. Alber et al., NA35 Collaboration, Z. Phys. C 66 (1995) 77

[19] N.M. Agababyan et al., NA22 Collaboration, Z. Phys. C 59 (1993) 195; C 66 (1995) 409; C 71 (1996) 405; Phys. Lett. B 422 (1998) 359

[20] See, e.g., the following reviews and references therein: $O$. Smirnova, Bose-Einstein correlations at the $Z$ peak, in: N.G. Antoniou et al. (Eds.), Proc. XXVIIIth Int. Symposium on Multiparticle Dynamics, World Scientific, Singapore, 1999, to be published; E.A. de Wolf, Bose-Einstein correlations, in: B.J. Pussey, I.G. Knowles (Eds.), Proc. XXVII Int. Conf. on H.E.P., Glasgow, Inst. of Phys. Publ., 1995, p. 1281; S. Haywood, Where are we going with Bose-Einstein - a mini review, RAL report RAL-94-074.

[21] B. Lörstad, O.G. Smirnova, Transverse Mass Dependence of Bose-Einstein Correlation Radii in $\mathrm{e}^{+} \mathrm{e}^{-}$annihilation at LEPEnergies, in: R.C. Hwa et al. (Eds.), Proc. 7th Int. Workshop on Multiparticle Production Correlations and Fluctuations, World Scientific, Singapore, 1997, p. 42.

[22] L3 Collaboration, B. Adeva et al., Nucl. Instr. Meth. A 289 (1990) 35; J.A. Bakken et al., Nucl. Instr. Meth. A 275 (1989) 81; O. Adriani et al., Nucl. Instr. Meth. A 302 (1991) 53; B. Adeva et al., Nucl. Instr. Meth. A 323 (1992) 109; K. Deiters et al., Nucl. Instr. Meth. A 323 (1992) 162; B. Acciari et al., Nucl. Instr. Meth. A 351 (1994) 300.

[23] T. Sjöstrand, Comp. Phys. Comm. 82 (1994) 74.

[24] The L3 detector simulation is based on GEANT3, see R. Brun et al., CERN report CERN DD/EE/84-1 (Revised), 1987, and uses GHEISHA to simulate hadronic interactions, see $\mathrm{H}$ Fesefeldt, RWTH Aachen report PITHA 85/02, 1985.

[25] G. Marchesini, B.R. Webber, Nucl. Phys. B 310 (1988) 461; G. Marchesini et al., Comp. Phys. Comm. 67 (1992) 465.

[26] S. Chapman, P. Scotto, U. Heinz, Phys. Rev. Lett. 74 (1995) 4400; Heavy Ion Physics 1 (1995) 1; S. Chapman, J.R. Nix, U. Heinz, Phys. Rev. C 52 (1995) 2694. 
[27] S. Hegyi, T. Csörgö, Proc. Budapest Workshop on Relativistic Heavy Ion Collisions, Preprint KFKI-1993-11/A, p. 47; T. Csörgo, in: A. Bialas et al. (Eds.), Proc. Cracow Workshop on Multiparticle Production, World Scientific, Singapore, 1994, p. 175.
[28] F.Y. Edgeworth, Trans. Cambridge Phil. Soc. 20 (1905) 36; see also, e.g., Harald Cramér, Mathematical Methods of Statistics, Princeton Univ. Press, 1946.

[29] K. Geiger, Comp. Phys. Comm. 104 (1997) 70. 